\title{
STRATÉGIE DE CHOIX D'UN FORMALISME DE FUSION MULTI-SOURCES ET APPORTS AU NIVEAU DU MODĖLE DE FUSION PAR LA DSMT : APPLICATION À LA CLASSIFICATION D'IMAGES SATELLITAIRES HAUTE RÉSOLUTION
}

\author{
Fatma Haouas ${ }^{1}$, Zouhour Ben Dhiaf ${ }^{1}$ \\ 1: Laboratoire LIPAH, FST Campus Universitaire, 2092 El Manar, Tunis, Tunisie \\ e-mail : fatmahaouas@hotmail.com, zouhour_bendhiaf@yahoo.fr
}

\begin{abstract}
Résumé
Nous présentons dans cet article, en premier lieu, une étude comparative de trois théories mathématiques permettant de réaliser la fusion des sources d'information. Cette étude vise à dégager les caractéristiques inhérentes aux théories des possibilités, des fonctions de croyance (TFC) et du raisonnement plausible et paradoxal (DSmT) afin d'établir une stratégie de choix qui nous permet d'adopter la théorie la plus adéquate pour résoudre un problème de fusion en tenant compte des informations acquises et des imperfections qui les accompagnent. En deuxième lieu, nous présentons également une nouvelle méthode pour déterminer les éléments focaux paradoxaux pertinents à l'aide des histogrammes de niveaux de gris et qui permet aussi de réduire la cardinalité de l'hyper-puissance. Ainsi, nous avons élaboré une nouvelle technique pour ordonner et coder les éléments focaux généralisés. Cette méthode est exploitée, en particulier, pour calculer la cardinalité de Dezert et Smarandache.
\end{abstract}

Mots clés : Fusion, Théorie des possibilités, Théorie des fonctions de croyance, Théorie du raisonnement plausible et paradoxal, Stratégie du choix, Imperfections d'information, éléments focaux paradoxaux, Cardinalité de DSm.

\begin{abstract}
We present in this paper, first, a comparative study of three mathematical theories to perform the fusion of information sources. This study aims to identify the inherent characteristics of the theories of possibilities, belief functions and plausible and paradoxical reasoning to establish a strategy of choice that allows us to adopt the most appropriate theory to solve a problem of fusion. This strategy is based on information and imperfections managed by each theory. Second, we present in this paper a new method to construct pertinent paradoxical classes based on gray levels histograms, which allows also the reduction of the cardinality of the hyper-powerset. Thus, we developed a new technique for ordering and coding generalized focal elements. This technique is exploited, in particular, to calculate the cardinality of Dezert and Smarandache.
\end{abstract}

Keywords : Fusion, Theory of possibilities, Theory of belief functions, Theory of plausible and paradoxical reasoning, Strategy of choice, Information imperfections, Paradoxical focal elements, DSm cardinality.

\section{Introduction}

La fusion d'information multi-source est largement utilisée dans plusieurs domaines tels que l'intelligence artificielle, la robotique, la fouille de données et la télédétection (Roux and Desachy, 1995; Milisavljevic and Bloch, 2003; Li et al., 2006; Alsun et al., 2010). Cette méthode de traitement d'information a commencé à attirer l'attention des chercheurs depuis ces deux dernières décennies ce qui a permis de mieux la comprendre, de l'appliquer et par la suite d'atteindre un énorme progrès dans cette branche d'étude. Cependant, appliquer la fusion nécessite une parfaite maîtrise des notions et des terminologies incontournables liées à cette branche : d'un côté la fiabilité et la disponibilité des informations, c'est-à-dire les imperfections qui les accompagnent telles que l'incertitude et l'imprécision, et d'un autre côté les approches et les algorithmes qui permettent de fusionner les sources (Bloch, 2005; Khaleghi et al., 2011). Parmi ces dernières, on trouve l'approche bayésienne qui représente un bon formalisme pour traiter l'incertitude. Les théories de l'incertain permettent d'appréhender de façon privilégiée les imperfections d'informations. Ainsi elles ont été étudiées et comparées dans plusieurs travaux (Parsons and Hunter, 1998; Martin, 2008). Par ailleurs, les travaux récents sur la théorie des fonctions de croyance ont donné naissance à une nouvelle extension nommée théorie du raisonnement plausible et paradoxal (Dezert, 2002). Par conséquent, il semble y avoir un besoin pour une étude analytique et comparative de l'évolution récente dans le domaine de fusion de données. Ce travail met l'accent sur trois théories de l'incertain qui sont les théories des possibilités, des fonctions de croyance et du raisonnement plausible et paradoxal, dans le but de pou- 
voir choisir la théorie la plus convenable à un problème donné. Ces approches ont démontré leur efficacité pour résoudre plusieurs problèmes de divers domaines. Néanmoins, les théories des possibilités et des fonctions de croyance ont échoué dans d'autres problèmes (Zadeh, 1984; Borotschnig et al., 1999; Lefevre et al., 2002; Liu, 2007; Florea et al., 2009; Tchamova and Dezert, 2012).

Afin d'établir une stratégie de choix d'un formalisme de fusion, nous avons focalisé la comparaison autour des informations et des formes d'imperfections qui les accompagnent qui peuvent être manipulées par chaque théorie.

\section{Fusion d'information multi-sources}

Plusieurs définitions ont été attribuées à la fusion d'information. Isabelle Bloch propose une définition générale qui englobe une grande variété des problèmes : "La fusion d'informations consiste à combiner des informations hétérogènes issues de plusieurs sources afin d'améliorer la prise de décision" (Bloch and Maître, 2004). Le groupe de travail européen FUSION (de 1996 à 1999) définit la fusion aussi dans un cadre générale : "La fusion d'informations consiste à regrouper des informations issues de plusieurs sources d'informations et à exploiter l'information regroupée". En d'autres termes, la fusion vise à exploiter les informations disponibles pour aboutir à une meilleure lecture et compréhension du phénomène traité en passant par plusieurs étapes primordiales et en utilisant diverses techniques.

La fusion peut s'appliquer pour résoudre une multitude de problèmes complexes grâce à son pouvoir de combinaison des sources de différentes natures et relations (Destercke et al., 2006; Milisavljevic and Bloch, 2003; Martin, 2008).

\subsection{Les étapes de la fusion}

La fusion est une tâche compliquée, par conséquent on a besoin de diviser ce processus en quelques étapes pour faciliter la tâche. Le modèle de fusion qui est généralement utilisé se déroule sur quatre étapes cruciales qui sont (Bloch and Maître, 2004; Martin, 2005) :

- La modélisation : Dans une étape préliminaire, il faut d'abord choisir un formalisme de fusion par suite définir le référentiel de travail.

- L'estimation : c'est une étape difficile vu qu'on ne dispose pas d'une méthode mathématique permettant d'obtenir le meilleur résultat. Elle consiste à affecter des valeurs numériques aux hypothèses.

- La combinaison : elle consiste à choisir un opérateur ou bien une règle de combinaison qui s'adapte le mieux au problème. Elle vise à regrouper des informations acquises pour avoir une information plus consistante et robuste.

- La décision : consiste à choisir une règle de décision afin de passer de l'information combinée vers le résultat.

\subsection{Les informations et les formes d'imperfection}

\subsubsection{Les informations}

Une information est une collection de symboles ou de signes produits soit par l'observation de phénomènes naturels ou artificiels, soit par l'activité cognitive humaine.

On distingue deux sortes d'information : objective et subjective. Les informations objectives sont issues de l'observation directe des phénomènes comme les mesures de capteurs. Tandis que celles dites subjectives sont les informations exprimées par des individus et conçues sans le recours à l'observation directe du monde réel.

L'information peut prendre deux formes : numérique et symbolique. Les informations numériques (généralement objectives) peuvent prendre différentes formes : nombres, intervalles de nombres... Les informations symboliques (subjectives) sont exprimées en langage naturel. Néanmoins, une information subjective peut être numérique et l'information objective peut être symbolique (Bloch, 2005; Dubois and Prade, 2009).

\subsubsection{Les imperfections de l'information}

Les informations acquises sont souvent entachées par plusieurs formes d'imperfection qui sont généralement dues aux phénomènes observés, aux limites des capteurs, au bruit, à la modélisation du problème ou bien au manque de fiabilité etc. (Smets, 1997; Dubois and Prade, 2009).

Les formes les plus difficiles à manipuler sont :

- L'incertitude : décrit la connaissance partielle et incomplète de la réalité. Elle traduit le degré de conformité de l'information avec le monde, en conséquence l'incertitude est un défaut quantitatif (Dubois and Prade, 2009). II faut mentionner qu'elle peut être de deux types. L'incertitude est dite épistémique lorsqu'elle est due au manque d'information acquise. Par ailleurs, elle est dite aléatoire si elle est due aux phénomènes aléatoires.

- L'imprécision : c'est un défaut qualitatif de l'information puisqu'elle décrit le manque d'exactitude de données. Cette imperfection est liée au contenu de l'information car elle est essentiellement une propriété de l'information elle-même.

- L'ambiguïté : cette forme d'imperfection apparaît lorsqu'on dispose de deux ou plusieurs interprétations de la même information.

- L'incomplétude : c'est le manque d'information apportée par la source.

- Le conflit : c'est l'imperfection la plus difficile à détecter et à gérer. Elle est souvent confondue avec d'autres d'imperfections. C'est la contradiction et l'inconsistance entre les informations acquises. Les origines du conflit proviennent essentiellement soit de la non fiabilité des sources, soit d'information erronée. Le conflit est engendré aussi lorsque les sources décrivent des phénomènes différents 
ou suite à une mauvaise modélisation du problème.

L'incertitude est l'imperfection la plus étudiée néanmoins, elle est toujours accompagnée par l'imprécision. Par ailleurs, l'incomplétude peut être une cause de l'apparition d'incertitude et d'imprécision.

II existe d'autres types d'imperfection tels que "vague" qui est une forme d'imprécision. Ce type est dû spécialement à la limitation du vocabulaire et dans la plupart des cas aux informations symboliques.

L'un des défis de la fusion de données multi-source est de réduire et d'éliminer les imperfections en exploitant la complémentarité et la redondance entre les sources d'information et par la suite atteindre l'information certaine, précise et robuste.

\section{Les formalismes de fusion}

\subsection{La théorie des possibilités}

La théorie des possibilités est induite de l'approche des sous-ensembles flous par Zadeh $(1968,1978)$ et est développée essentiellement en France par Dubois and Prade $(1985,2003)$. Elle est connue pour sa simplicité et elle représente un cadre théorique formel non probabiliste pour traiter les informations hétérogènes et incomplètes. Par conséquent, elle modélise l'imprécision et l'incertitude. Elle se base sur la fonction de distribution de possibilité $\pi$ qui permet d'associer à chaque hypothèse de l'univers du discours $\Omega=\left\{\omega_{1}, \ldots, \omega_{n}\right\}$ un degré de possibilité dans $[0,1]$ et qui vérifie l'équation suivante :

$$
\sup _{\omega \in \Omega}\{\pi(\omega)\}=1 .
$$

Cette expression traduit l'existence d'au moins une hypothèse de l'univers du discours qui est complètement possible. Étant donné $x$ une observation, $\pi_{x}(\omega)$ désigne le degré de possibilité pour que $x=\omega$ :

- $\pi_{x}(\omega)=1$, c'est-à-dire il est complètement possible que $x=\omega$.

- $\pi_{x}(\omega)=0$, c'est-à-dire il est impossible que $x=\omega$.

La théorie des possibilités utilise deux fonctions duales pour quantifier l'imprécision et l'incertitude qui sont respectivement les fonctions mesure de possibilité $\Pi$ et mesure de nécessité $N$. Elles sont calculées à l'aide de la fonction de distribution de possibilité.

La mesure de possibilité $\Pi$ est une fonction de $2^{\Omega}$ (l'ensemble des parties de $\Omega$ ) à valeur dans $[0,1]$ et qui vérifie le système suivant :

$$
\begin{cases}\Pi(\Omega) & =1 \\ \forall A \in 2^{\Omega}, \Pi(A) & =\sup \{\pi(x), x \in A\}\end{cases}
$$

Où $\emptyset$ est l'ensemble vide.

La fonction de mesure de nécessité $N$ est donnée par les équations suivantes:

$$
\begin{cases}N(\Omega) & =1 \\ \forall A \in 2^{\Omega}, N(A) & =\inf \{1-\pi(x), x \notin A\}\end{cases}
$$

L'un des intérêts majeurs de la fusion par la théorie des possibilités est la grande variété d'opérateurs de fusion (combinaison). Le grand nombre d'opérateurs a motivé la communauté à faire un classement de ceux-ci afin de permettre le choix de l'opérateur le plus adéquat à l'application. Ce choix peut se faire selon plusieurs critères liés aux propriétés des opérateurs (et aux natures des informations) (Bloch, 1996). II est courant de considérer les opérateurs conjonctifs (exemple : $t$ normes) et disjonctifs (exemple : t-conormes). II existe d'autres catégories telles que les opérateurs de moyennes ou de compromis.

Les opérateurs conjonctifs ont le comportement d'un "ET logique". Ainsi, le résultat sera proche de 1 si tous les degrés de possibilité à combiner le sont aussi. Parmi lesquelles on trouve la t-norme de Zadeh:

$$
\pi(\omega)=\min \left(\pi^{S_{1}}(\omega), \pi^{S_{2}}(\omega)\right) .
$$

Les opérateurs disjonctifs combinent les informations à la façon d'un "OU logique". Ces opérateurs augmentent l'imprécision et par suite la certitude ainsi ils ont un comportement indulgent. Ils sont appliqués lorsque les sources sont disjonctives. Nous citons par exemple la tconorme de Zadeh :

$$
\pi(\omega)=\max \left(\pi^{S_{1}}(\omega), \pi^{S_{2}}(\omega)\right) .
$$

Les opérateurs adaptatifs ont un comportement de compromis. Ils s'adaptent en fonction du conflit entre les sources. Par exemple l'opérateur de Dubois and Prade (1994b) se comporte comme un minimum s'il y a peu de conflit entre les sources et comme un maximum si le conflit est élevé. En effet, Il est lié à un consensus $h$ (mesure du conflit) entre les différentes distributions de possibilités, à savoir la borne supérieure de leurs intersections :

$$
\left\{\begin{array}{l}
h=\max _{\omega}\left(\min \left(\pi^{S_{1}}(\omega), \pi^{S_{2}}(\omega)\right)\right) \\
\pi(\omega)=\max \left(\frac{\min \left(\pi^{S_{1}}(\omega), \pi^{S_{2}}(\omega)\right)}{h}\right. \\
\left.\min \left(\max \left(\pi^{S_{1}}(\omega), \pi^{S_{2}}(\omega)\right), 1-h\right)\right)
\end{array}\right.
$$

La décision au sein de la théorie des possibilités se fait habituellement par le critère de maximum de possibilité. On choisit la décision $d_{k}$ si :

$$
\pi^{k}(x)=\max \left(\pi^{i}(x), i=1 . . n\right) .
$$

\subsection{La théorie des fonctions de croyance}

La théorie de l'évidence connue aussi par la théorie de Dempster Shafer (la DST) ou des fonctions de croyance (la TFC) a été mise en place par Shafer (Shafer, 1976, 1990; Vannoorenberghe, 2003). Elle constitue un formalisme probabiliste permettant de traiter la méconnaissance qu'elle soit partielle ou totale, l'incertitude et l'imprécision. L'ensemble de toutes les hypothèses nécessaires pour décrire le problème de fusion s'appelle cadre de discernement $\Omega$ qui doit être exhaustif et exclusif. Contrairement à la théorie des possibilités, le raisonnement ici porte sur $2^{\Omega}$ qui représente l'ensemble 
de toutes les parties (les sous-ensembles) du cadre de discernement et qui est appelé l'ensemble de puissance.

La fonction fondamentale de la TFC s'appelle fonction de masse notée $m($.). Elle permet d'associer à chaque élément de $2^{\Omega}$ un degré de confiance dans $[0,1]$ et qui vérifie le système d'équations suivant :

$$
\begin{cases}m(\Omega) & =1 \\ \sum_{A \in 2^{\Omega}} m(A) & =1\end{cases}
$$

Les entités de masse non nulles s'appellent éléments focaux. Une proposition $B \in 2^{\Omega}$, par exemple $B=\omega_{1} \cup \omega_{2}$, représente explicitement le doute entre les hypothèses composant $B$ mais la masse de croyance $m(B)$ allouée à $B$ ne donne aucune information sur les hypothèses et les sous-ensembles formant $B$. Ainsi, les masses correspondantes aux hypothèses simples expriment la certitude d'une hypothèse par rapport aux autres, alors que la masse des entités composées représente l'ignorance et l'incertitude.

Les fonctions de croyance $C r($.$) et de plausibilité$ $P l($.$) dérivées de celle de masse sont définies de 2^{\Omega}$ dans $[0,1]$ :

$$
\forall A \in 2^{\Omega},\left\{\begin{array}{l}
C r(A)=\sum_{B \subseteq A} m(B) \\
\operatorname{Pl}(A)=\sum_{B \cap A \neq \emptyset} m(B)
\end{array}\right.
$$

$\operatorname{Cr}(A)$ est la confiance que la vérité soit dans $A$. Par contre $P l(A)$ dénote la plausibilité que la vérité soit dans $A$. La croyance et la plausibilité représentent respectivement l'incertitude minimale et maximale.

Pour combiner les masses de l'évidence, Dempster a mis en place l'opérateur de somme orthogonale appelé aussi la règle de Dempster qui est conjonctive et commutative (Shafer and Logan, 1987). Pour deux sources $S_{1}$ et $S_{2}$ cette règle s'écrit comme suit :

$$
\forall A \in 2^{\Omega}, m(A)=\sum_{B \cap C=A} m^{S_{1}}(B) * m^{S_{2}}(C) .
$$

$m^{S_{1}}$ et $m^{S_{2}}$ représentent les fonctions de masse respectives de $S_{1}$ et $S_{2}$. L'inconvénient de cette forme est que la masse du vide peut être différente de zéro ce qui est inacceptable puisque elle doit être égale à zéro. Cet inconvénient a poussé à normaliser la règle conjonctive comme suit :

$$
\begin{cases}k & =\sum_{C_{1} \cap C_{2}=\emptyset} m^{s_{1}}\left(C_{1}\right) * m^{s_{2}}\left(C_{2}\right) \\ \forall A \in 2^{\Omega}, m(A) & =\sum_{B \cap C=A} \frac{m^{s_{1}}(B) * m^{s_{2}}(C)}{1-k}\end{cases}
$$

$k$ est appelée masse du vide ou encore inconsistance de fusion. Elle quantifie le conflit entre les sources impliquées. Ainsi, la constante $k$ appartient à l'intervalle $[0,1]$. Lorsque $k$ est très grand (i.e. très proche de 1 ) les sources sont très conflictuelles. II existe d'autre règles de combinaison telles que la règle de Dubois et Prade et la règle de Yager (Martin, 2010).
La théorie des fonctions de croyance offre plusieurs règles de décision qui se fondent sur la maximisation d'un critère. Le choix de la règle de décision dépend généralement du problème abordé. Les plus utilisées sont :

- Maximum de crédibilité : ce critère consiste à choisir l'entité qui a la plus grande valeur de crédibilité. Ainsi, pour l'observation $x$ on décide $A_{i}$ si :

$$
C r\left(A_{i}\right)(x)=\max \left\{C r\left(A_{j}\right)(x), \quad 1 \leq j \leq\left|2^{\Omega}\right|\right\} .
$$

- Maximum de plausibilité : permet de choisir la décision qui a le maximum de plausibilité. Donc pour chaque observation donnée la décision se fait comme suit :

$$
P l\left(A_{i}\right)(x)=\max \left\{P l\left(A_{j}\right)(x), \quad 1 \leq j \leq\left|2^{\Omega}\right|\right\} .
$$

- Maximum de probabilité pignistique : La probabilité pignistique introduite par Smets (1990a). Elle répartit équitablement les masses placées sur des éléments différents d'un singleton sur les singletons qui les composent. Ainsi, pour chaque élément de $2^{\Omega}$ la probabilité se calcule comme suit :

$$
\operatorname{BetP}(A)=\sum_{B \in 2^{\Omega}, B \neq \emptyset} \frac{|A \cap B|}{|B|} m(B) .
$$

Cette règle revient à décider $\omega_{i}$ pour l'observation $x$ si :

$$
\operatorname{Bet} P\left(\omega_{i}\right)(x)=\max \left\{\operatorname{Bet} P\left(\omega_{i}\right)(x), i \in\{1 . .|\Omega|\}\right\} .
$$

\subsection{La théorie du raisonnement plausible et para- doxal}

Les travaux menés par Dezert et Smarandache sur la TFC ont eu pour but de surmonter ces limites inhérentes et ont donné la naissance de la nouvelle théorie du raisonnement plausible et paradoxal en 2001(Dezert, 2002; Dezert and Smarandache, 2005, 2009). Appelée aussi théorie du Dezert et Smarandache ou encore DSmT, elle permet de traiter le paradoxe entre les sources d'information et ceci en réfutant la contrainte d'exclusivité du cadre de discernement qui est modélisée par l'intersection des hypothèses. Ainsi, on parle du cadre de discernement généralisé et non plus de l'ensemble de puissance mais de l'hyper-puissance noté $D^{\Omega}$ qui renferme tous les sous-ensembles formés par l'opérateur d'union " $\cup$ " et celui d'intersection " $\cap$ ".

Exemple : Soit un cadre de discernement généralisé formé par deux hypothèses $\Omega=\left\{H_{1}, H_{2}\right\}$ donc l'hyperpuissance est $D^{\Omega}=\left\{\emptyset, H_{1}, H_{2}, H_{1} \cup H_{2}, H_{1} \cap H_{2}\right\}$.

La fonction de masse généralisée est donc définie sur $D^{\Omega}$ dans $[0,1]$ :

$$
\begin{cases}m(\emptyset) & =0 \\ \sum_{A \in D^{\Omega}} m(A) & =1\end{cases}
$$


Les fonctions de croyance et de plausibilité généralisées sont définis d'une manière analogue que dans la TFC.

La règle de combinaison de Dezert-Smarandache classique (la DSmC) n'est que la forme généralisée de l'opérateur de somme orthogonale. Pour deux sources distinctes $S_{1}$ et $S_{2}$, la DSmC s'écrit :

$$
\forall A \in D^{\Omega}, m(A)=\sum_{B \cap C=A} m^{S_{1}}(B) * m^{S_{2}}(C) .
$$

Une diversité de règles de combinaison a été mise en place pour combiner les masses de croyances généralisées telles les cinq versions de PCR (Proportional Conflict Redistribution) (Smarandache and Dezert, 2005a,b). L'idée consiste à redistribuer le conflit qu'il soit partiel ou total sur les entités qui génèrent le conflit. La plus utilisée et la plus sophistiquée mathématiquement est la cinquième version notée PCR5 (Smarandache et al., 2010). II existe d'autres règles telles que la $\mathrm{DSmH}$ (la règle de DSm Hybride), URR, PURR MURR que nous n'allons pas détailler dans cet article (Dezert and Smarandache, 2009).

La DSmT offre trois modèles de fusion qui sont les modèles libre, hybride et de Shafer.

- Modèle libre : Noté $M^{F}$ il représente le problème où l'ensemble $D^{\Omega}$ renferme toutes les combinaisons possibles construites à partir de $\Omega$ à l'aide des opérateurs $\cup$ et $\cap$. Ce modèle entraine un grand nombre d'entités composées, parmi lesquelles il existe des éléments de masses nulles ou non significatifs.

- Modèle hybride : Noté $M$, il résulte de l'introduction des contraintes d'exclusivité et de non existence et mêmes mixtes. Ainsi, plusieurs combinaisons ne figurent pas dans $D^{\Omega}$. En conséquence, ce modèle est moins coûteux que le premier.

- Modèle de Shafer : On obtient ce modèle $M^{0} \mathrm{si}$ toutes les contraintes d'exclusivité sont imposées et dans ce cas on se trouve dans le cadre de la TFC.

Tout comme la TFC, la DSmT offre plusieurs règles de décision. Cependant, pour utiliser le critère de décision par la probabilité pignistique il faut généraliser la règle proposée par Smets puisque celle-ci ne peut pas être appliquée avec n'importe quel jeu de masse généralisé issu de la phase de combinaison. Dans le même ordre d'idées, Dezert et al. (2004) proposent une transformation pignistique généralisée qui s'adapte avec ce nouveau cadre théorique. Ainsi, $\forall A_{i} \in D^{\Omega}$ :

$$
\operatorname{GPT}\left(A_{i}\right)=\sum_{A_{j} \in D^{\Omega}} \frac{C_{M}\left(A_{i} \cap A_{j}\right)}{C_{M}\left(A_{j}\right)} * m\left(A_{j}\right) .
$$

où $C_{M}\left(A_{i}\right)$ représente la cardinalité de DSm. C'est une nouvelle notion de cardinalité importante pour pouvoir calculer la transformation pignistique généralisée. La cardinalité de DSm de n'importe quel élément $A$ de l'hyper-puissance notée $C_{M}(A)$ est le nombre de parties de cet élément dans le diagramme de Venn qui correspond au problème $M$.

\section{Stratégie de choix d'un formalisme de fusion}

\subsection{Comparaison des approches}

La théorie des possibilités se dérive du fondement flou, cependant les théories des fonctions de croyance et du raisonnement plausible et paradoxal découlent d'une approche probabiliste. En outre, il est clair que ces approches n'ont pas les mêmes capacités de modélisation ni de raisonnement. Ainsi, il est certain que les objets mathématiques manipulés sont différents.

Par contre, la théorie des possibilités devient un cas particulier de la TFC lorsque les éléments focaux sont emboîtés (Dubois and Prade, 1994a). En effet, on note par $F$ l'ensemble des éléments focaux d'un problème tel que $F=F_{1}, F_{2}, . ., F_{n}$ si $F_{1} \subseteq F_{2} \subseteq \ldots \subseteq F_{n}$ on a alors :

$$
\begin{aligned}
& C r(A \cap B)=\min (C r(A), C r(B)) \text { et } \\
& \operatorname{Pl}(A \cup B)=\max (\operatorname{Pl}(A), P l(B))
\end{aligned}
$$

Par ailleurs, le troisième modèle de DSm (modèle de Shafer) représente la TFC.

La théorie des possibilités offre un cadre de modélisation simple qui permet de fusionner des univers du discours continus (Zadeh, 1978; Dubois and Prade, 1994a). En revanche, le cadre adéquat de l'application de la TFC et la DSmT est l'univers discret.

La méthode de raffinement/grossissement donne la possibilité à la TFC d'unifier et fusionner les cadres de discernement différents (Appriou, 2005). Or la théorie des possibilités semble avoir besoin d'une technique qui lui permet de fusionner des sources ayant des univers de discours différents mais complémentaires.

Bien que les théories des possibilités et des fonctions de croyance exigent l'exclusivité et l'exhaustivité du référentiel de définition, la modélisation au sein de la TFC est plus souple et riche que celle du formalisme possibiliste vu qu'elle ne travaille pas uniquement sur des singletons mais aussi sur des disjonctions. La DSmT s'est affranchie de la contrainte d'exclusivité du cadre de discernement ce qui lui a donné la capacité de traiter le paradoxe entre les sources d'évidence et par la suite modéliser des problèmes où la TFC n'en est pas capable.

L'approche possibliste estime la distribution de possibilité uniquement sur des singletons alors que la TFC porte son raisonnement sur $2^{\Omega}$ et la DSmT opère sur $D^{\Omega}$ ce qui signifie que la théorie des possibilités est la moins coûteuse par rapport aux deux autres. Dans ce même ordre d'idée, la DSmT engendre une complexité exponentielle beaucoup plus élevée que celle de la TFC (exemple : si $|\Omega|=4$ alors $\left|2^{\Omega}\right|=16$ et $\left|D^{\Omega}\right|=167$ ). En conséquence, il devient d'une extrême difficulté de fusionner des sources donnant des informations sur des cadres de discernement généralisés de cardinalité supérieure ou 
égale à 6 , surtout lorsque le modèle de fusion appliqué est le modèle libre (Dezert and Smarandache, 2003). De plus, cette explosion d'hypothèses composées engendre la présence d'entités non significatives dans l'hyperpuissance.

La DSmT offre des règles de combinaison puissantes et meilleures que celles données par la TFC. Citons la règle PCR5 "Proportional Conflict Redistribution" qui tient compte du conflit et qui est plus sophistiquée et plus puissante que la règle de Dempster normalisée (Smarandache and Dezert, 2006). Par ailleurs, la règle $\mathrm{DSmH}$ (règle de combinaison hybride) est adaptée dans la fusion dynamique tandis que la règle de Dubois et Prade (la règle de combinaison mixte au sein de la TFC) ne peut être adaptée dans ce même cas sachant que la $\mathrm{DSmH}$ est une extension de la règle de Dubois et Prade. Dans le même ordre d'idees la DSmC permet de combiner des fonctions de masse hautement conflictuelles contrairement à la règle de Dempster normalisée. Cependant, les règles de combinaisons au sein des deux formalismes engendrent un très grand nombre d'itérations ce qui augmente la complexité temporelle dont elles souffres les deux théories.

D'autre part, la richesse combinatoire au sein de l'approche possibiliste constitue un avantage certain, néanmoins le choix de l'opérateur adéquat devient une tâche difficile même si les opérateurs offerts par ce formalisme sont répertoriés dans plusieurs catégories.

Un autre intérêt de la TFC réside dans sa décision riche grâce à la variété de critères de décision. C'est le cas aussi pour la DSmT. Toutefois celle-ci ne nous offre pas une règle de décision qui nous permet d'avoir un résultat formé uniquement par les hypothèses du cadre de discernement généralisé.

\subsection{Stratégie de choix proposée}

Face au problème de fusion la première tâche consiste à choisir un formalisme qui s'adapte le mieux au problème abordé (Smets, 1998; Ben Amor and Martel, 2004; Dubois and Prade, 2009).

La théorie des possibilités est un cadre théorique pour la représentation des données imprécises que se soient symboliques ou numériques et d'informations pauvres (incomplètes). Cette dernière permet de représenter les objets dont les critères d'appartenance sont graduels comme elle permet de manipuler les informations symboliques et les informations exprimées en langage naturel. Ainsi, cette théorie possède le formalisme approprié pour fusionner les informations expertes. En revanche, la théorie des possibilités permet la manipulation des informations spatiales (Bloch, 2005) et de l'incertitude épistémique et non pas aléatoire (Dubois and Prade, 2009). II est vrai que les opérateurs disjonctifs et adaptatifs ont permis de combiner des distributions conflictuelles, mais ils ont perdu le défi devant les sources hautement conflictuelles.

D'un autre coté, la TFC permet de manipuler l'imprécision, les deux types d'incertitudes (aléatoires et épistémiques) et l'ambiguïté. Par ailleurs, si les sources ne sont pas fiables il vaut mieux choisir le formalisme évidentiel (TFC) pour fusionner les informations fournies par ces sources. La gestion du conflit au sein de la TFC peut se faire à l'aide de plusieurs méthodes telles que la normalisation de la règle de Dempster, la proposition de d'autres règles de combinaison et l'affaiblissement des sources non fiables (Shafer, 1976). De plus, une extension de cette théorie a été proposée par Smets (1990b) permettant de gérer le conflit entre les sources qui s'appelle "Modèle de Croyance Transférable (MCT)" qui permet de travailler dans un cadre de discernement non exhaustif. Cependant, ces méthodes restent incapables de fusionner des sources hautement conflictuelles dans certains cas.

Toutefois, la théorie du raisonnement plausible et paradoxal permet de manipuler l'imprécision, les incertitudes épistémiques et aléatoires, l'ambiguïté et surtout le paradoxe entre les sources d'évidence sur des univers discrets (Smarandache and Dezert, 2005a). Ainsi, cette théorie manipule la majorité des formes d'imperfection qui accompagnent les informations et fusionne des sources ayant des fiabilités très différentes.

La figure suivante représente une stratégie de choix d'un formalisme de fusion selon les informations et les imperfections qui les accompagnent :

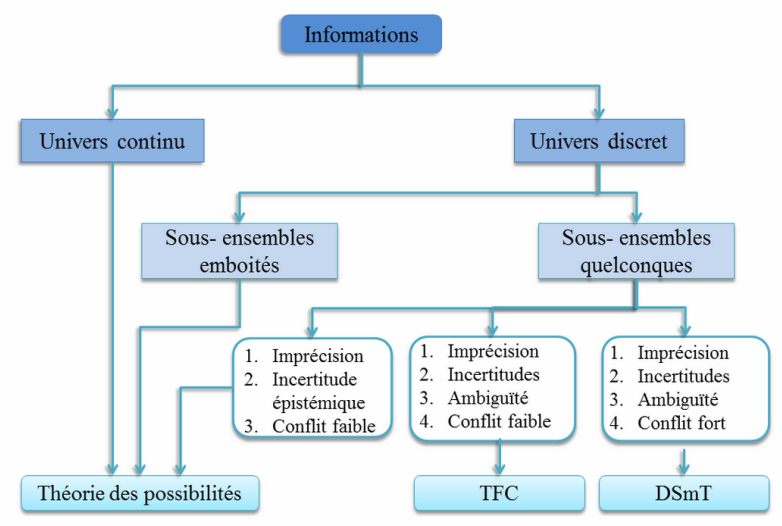

FIGURE 1 : Stratégie de choix d'un formalisme de fusion.

\section{Méthode proposée pour générer des classes paradoxales}

Pour appliquer la DSmT, nous devons tout d'abord déterminer et générer les différentes classes composées dont le nombre est généralement élevé.

Par contre, il faut souligner que les entités composées ont des significations sémantiques bien déterminées. En effet, les classes composées par l'opérateur d'union traduit l'incertitude alors que les classes formées par la conjonction des singletons représentent le paradoxe.

Ainsi, il semble nécessaire de trouver une méthode pour 
les déterminer en respectant leur signification.

\section{Exemple :}

Soit un cadre de discernement généralisé formé par deux classes non exclusives $\Omega=\left\{C_{1}, C_{2}\right\}$, alors: $m\left(C_{1}\right)+m\left(C_{2}\right)+m\left(C_{1} \cup C_{2}\right)+m\left(C_{1} \cap C_{2}\right)=1$.

Donc $m\left(C_{1} \cup C_{2}\right)$ représente l'incertitude et l'absence de l'information pour décider entre $C_{1}$ et $C_{2}$. Par contre, $m\left(C_{1} \cap C_{2}\right)$ est la masse paradoxale. Elle traduit l'inconsistance et le conflit entre les deux classes.

Pour s'affranchir de cette difficulté, nous avons utilisé une méthode proposée par Ben Dhiaf (2011) pour construire les éléments focaux de type classe incertaine. Cette méthode se repose sur les histogrammes de niveaux de gris des classes.

L'histogramme d'une classe incertaine $A$ (formée par la disjonction des hypothèses) est formé par les bornes inférieurs des histogrammes des classes incluses dans la classe $A$ (fréquences minimales). Sachant que l'auteur considère qu'une classe $C$ est un élément focal pour un niveau de gris $x$ si sa fonction histogramme $h_{C}$ est non nulle pour $x$.

Ainsi, le nombre des classes composées par l'union est réduit.

Nous avons généralisé cette approche en proposant une nouvelle méthode pour générer les classes paradoxales ( classes formées par l'opérateur d'intersection). En effet, nous proposons de mieux exploiter les zones des chevauchements des histogrammes de niveaux de gris puisqu'elles représentent non seulement l'incertitude mais aussi l'ambiguïté et le conflit.

Pour déterminer l'histogramme d'une classe paradoxale $B$ nous avons considéré les fréquences maximales de la zone de chevauchements des histogrammes $h_{C_{i}}$ des classes $C_{i}$ qui forment par leurs intersections la classe $B$ où $h_{C_{i}}$ représente l'histogramme relatif à la classe $C_{i}$.

Ainsi, l'histogramme de la classe $B$ est donné par l'équation suivante :

$h_{B}(x)=\max \left\{h_{C h}(x) \mid C_{i} \cap B=B, \quad i \in\{1 . . K\}\right\}$.

Sachant que $h_{C h}$ est la fonction histogramme de la zone de chevauchements des classes qui composent par leurs intersections la classe $B$.

\section{Exemple :}

Étant donné un cadre de discernement généralisé formé par trois classes non mutuellement exclusives $\Omega=$ $\left\{C_{1}, C_{2}, C_{3}\right\}$, telle que $C_{1} \cap C_{3} \equiv \emptyset$. Leurs histogrammes de niveaux de gris sont illustrés par la figure 2 .

Considérant une zone de chevauchement de $C_{1}$ et $C_{2}$, l'histogramme de la classe $C_{1} \cap C_{2}$ est formé par les fréquences maximales de cette zone considérée. Or, ces fréquences correspondent à l'histogramme de $C_{2}$. Au lieu d'estimer la fonction de masse sur un ensemble formé par 19 éléments nous sommes parvenu à l'estimer sur 7 éléments focaux pertinents.

De cette manière nous avons exprimé le paradoxe entre les classes, et déterminé les éléments focaux

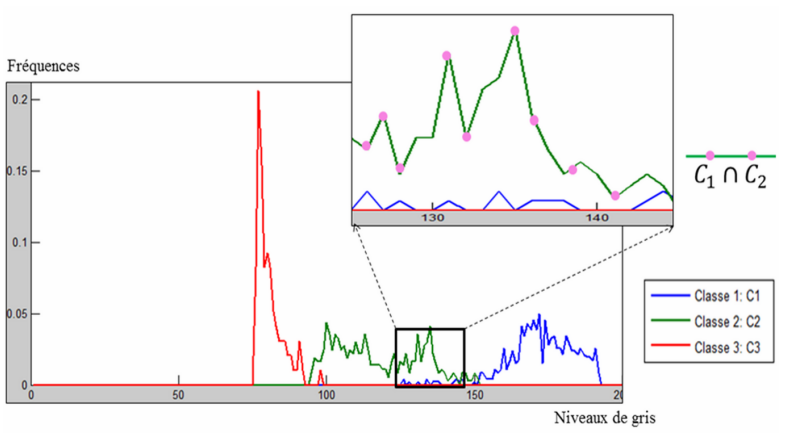

FIGURE 2 : Génération de classes paradoxales.

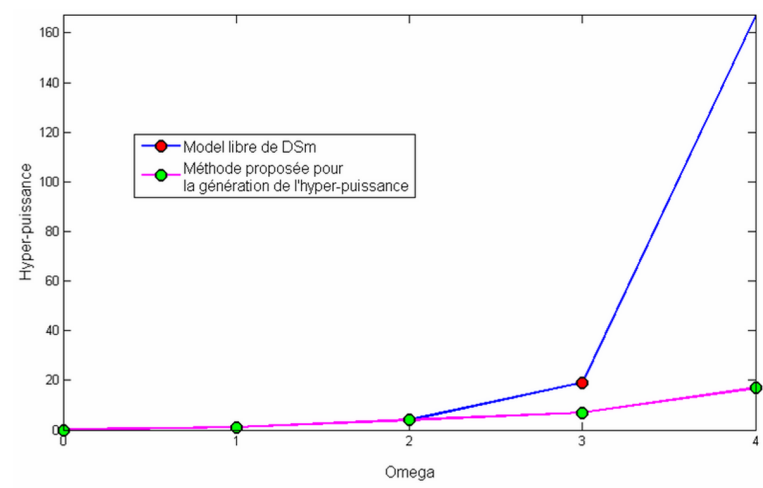

FIGURE 3 : Comparaison entre la cardinalité de $D^{\Omega}$ obtenu par le modèle libre et par notre méthode.

généralisés. Nous avons donc réduit la cardinalité de $D^{\Omega}$ en intégrant des contraintes d'exclusivité en fonction des histogrammes et en éliminant des classes composées non significatives ou de masse nulle.

\section{Nouvelle technique de codification et d'ordonnancement des éléments focaux généralisés}

La modélisation riche au sein de la DSmT engendre un grand nombre de classes composées. Parmi elles, il existe des classes formées par les mêmes hypothèses singletons mais les opérateurs sont différents. L'un des problèmes que l'on peut rencontrer, pendant la mise en place de la DSmT, est la distinction entre les classes composées par l'union et l'intersection des singletons (exemple : $C_{1} \cup C_{2}$ et $C_{1} \cap C_{2}$ ) ce qui engendre une ambiguïté au cours de la phase de combinaison. Nous avons besoin d'une technique permettant de gérer les différentes classes.

Pour résoudre ce problème, nous proposons de construire une matrice binaire qui contient les codes des éléments focaux généralisés en indiquant les classes qui les constituent et les opérateurs qui les forment. La matrice de codification est caractérisée par sa flexibilité puisque sa taille dépend du nombre des éléments focaux.

Le nombre de ses lignes est donc égal au nombre d'éléments focaux que nous notons $K_{\text {gen }}$. Ses colonnes correspondent aux $K$ classes singletons $(K=|\Omega|)$, classes 
d'union simples et classes d'intersection simples plus deux autres colonnes qui servent à indiquer les types d'opérateurs utilisés. Les lignes contiennent les codes des éléments focaux et les colonnes représentent les classes utilisées pour construire ces éléments. Ainsi, la présence de 1 dans une colonne donnée d'une ligne donnée indique soit l'utilisation de la classe de la colonne soit de l'opérateur de la colonne.

Cette matrice est ordonnée de sorte que les $K$ premières lignes correspondent aux singletons. Les lignes suivantes contiennent les classes d'union simple suivies des classes d'intersection simple et finalement les classes mixtes c-à-d les classes composées par les deux opérateurs.

Exemple : Soit $\Omega=C_{1}, C_{2}, C_{3}$ un cadre de discernement satisfaisant le modèle hybride, sachant que $C_{2} \cap$ $C_{3}=\emptyset . D^{\Omega}=\left\{C_{1}, C_{2}, C_{3}, C_{1} \cup C_{2}, C_{1} \cup C_{3}, C_{1} \cap C_{2}, C_{1} \cap\right.$ $\left.C_{3}\right\}$. Les éléments de l'hyper-puissance sont notés respectivement de $A_{1}$ jusqu'à $A_{7}$. La matrice relative à cet ensemble est :

\begin{tabular}{||c|c|c|c|c|c|c|c|c|c||}
\hline \hline & $A_{1}$ & $A_{2}$ & $A_{3}$ & $A_{4}$ & $A_{5}$ & $A_{6}$ & $A_{7}$ & $\cup$ & $\cap$ \\
\hline$A_{1}$ & 1 & 0 & 0 & 0 & 0 & 0 & 0 & 0 & 0 \\
\hline$A_{2}$ & 0 & 1 & 0 & 0 & 0 & 0 & 0 & 0 & 0 \\
\hline$A_{3}$ & 0 & 0 & 1 & 0 & 0 & 0 & 0 & 0 & 0 \\
\hline$A_{4}$ & 1 & 1 & 0 & 0 & 0 & 0 & 0 & 1 & 0 \\
\hline$A_{5}$ & 1 & 0 & 1 & 0 & 0 & 0 & 0 & 1 & 0 \\
\hline$A_{6}$ & 1 & 1 & 0 & 0 & 0 & 0 & 0 & 0 & 1 \\
\hline$A_{7}$ & 1 & 0 & 1 & 0 & 0 & 0 & 0 & 0 & 1 \\
\hline \hline
\end{tabular}

TABLE 1 : Exemple de matrice de codification des éléments de I'hyper-puissance.

Par exemple pour coder la classe $C_{1}$ qui est $A_{1}$ dans le tableau de codification il suffit de mettre un 1 dans la première colonne de la ligne de $C_{1}$.

$A_{4}=C_{1} \cup C_{2}$ est une classe incertaine (type union), pour la coder il suffit de mettre des 1 dans les colonnes correspondantes à $C_{1}, C_{2}\left(A_{1}\right.$ et $\left.A_{2}\right)$ et la colonne $\cup$.

Le code de la classe $A_{6}=C_{1} \cap C_{2}$ présente une légère différence avec $A_{4}=C_{1} \cup C_{2}$. Alors, on met un 1 dans la colonne $\cap$ et non pas $\cup$. Cet exemple présente le tableau de codification correspondant à un cadre de discernement qui satisfait le modèle hybride. Dans le cas où les hypothèses sont deux à deux non exclusives l'hyperpuissance est donc $D^{\Omega}=\left\{C_{1}, C_{2}, C_{3}, C_{1} \cup C_{2}, C_{1} \cup\right.$ $C_{3}, C_{2} \cup C_{3}, C_{1} \cup C_{2} \cup C_{3}, C_{1} \cap C_{2}, C_{1} \cap C_{3}, C_{2} \cap C_{3}, C_{1} \cap$ $C_{2} \cap C_{3}, C_{1} \cap\left(C_{2} \cup C_{3}\right), C_{2} \cap\left(C_{1} \cup C_{3}\right), C_{3} \cap\left(C_{1} \cup C_{2}\right), C_{1} \cup$ $\left.\left(C_{2} \cap C_{3}\right), C_{2} \cup\left(C_{1} \cap C_{3}\right), C_{3} \cup\left(C_{1} \cap C_{2}\right)\right\}$. La matrice de codification est donc formée par 17 lignes. Les colonnes représentent 11 classes de $C_{1}$ jusqu'à $C_{1} \cap C_{2} \cap C_{3}$ et les opérateurs d'union et d'intersection. A titre d'exemple, pour coder $C_{1} \cap\left(C_{2} \cup C_{3}\right)$ il faut mettre des 1 dans les colonnes correspondantes à $C_{1}, C_{2} \cup C_{3}$, $\cup$ et $\cap$.

\section{Méthode proposée pour calculer la cardinalité de DSm}

Le calcul de la transformation pignistique généralisée nécessite le calcul de la cardinalité de DSm. Pour cal- culer la cardinalité de DSm d'une classe de l'ensemble de l'hyper-puissance on doit avoir recours au diagramme de Venn qui représente le modèle choisi (libre, hybride ou bien de Shafer) et le problème abordé. Cependant, on ne dispose pas de formules qui nous permettent de calculer cette cardinalité quelque soit le modèle et le type de classe.

Afin d'éviter le calcul manuel de la cardinallité de DSm des classes, nous avons exploité la technique de codification et d'ordonnancement que nous avons mis en place pour proposer une méthode permettant de calculer automatiquement cette cardinalité.

Soient $K$ le nombre des classes singletons (cardinalité du cadre de discernement) et $K_{\text {nouv }}$ le nombre des classes singletons plus le nombre des classes d'union. Pour calculer la cardinalité des classes singletons, d'union simple et d'intersection simple, nous procédons comme suit :

- Calcul de la cardinalité d'une classe singleton $C_{i}$ :

1. Considérer les $K_{\text {nouv }}$ premières lignes de la colonne $C_{i}$ de la matrice de codification.

2. $C_{M}\left(C_{i}\right)=$ nombre de 1 dans la partie considérée de la colonne.

- Calcul de la cardinalité d'une classe d'union simple A :

1. Considérer la sous-matrice formée par les $K_{\text {nouv }}$ premières lignes de la matrice de codification.

2. Les colonnes de la sous-matrice correspondent aux classes incluses dans $A$.

3. $C_{M}(A)=$ nombre de lignes qui contiennent au moins un 1.

- Calcul de la cardinalité d'une classe d'intersection simple $B$ :

1. Considérer la sous-matrice formée par les lignes de codes des classes d'intersection simple.

2. Les colonnes correspondent aux classes qui forment $B$

3. $C_{M}(B)=$ nombre de lignes qui contiennent que des 1.

Exemple : Reprenons l'exemple de la section précédente. Le diagramme de Venn de ce cadre est illustré par la figure 4.

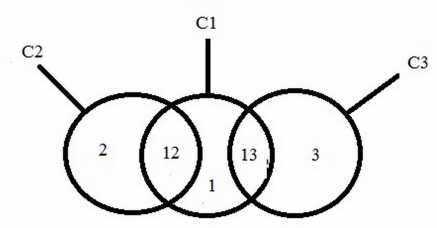

FIGURE 4 : Diagramme de Venn. 


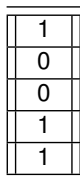

TABLE 2 : Cardinalité de $C_{1}$.

Pour calculer la cardinalité de $C_{1}$ qui est égale à 3 , nous considérons les 5 premières lignes de colonne $C_{1}$ dans la matrice de codification $\left(5=K_{\text {nouv }}\right)$.

Ainsi, la cardinalité de $C_{1}$ est égale au nombre de 1 dans cette colonne qui est 3 .

Pour calculer la cardinalité de $C_{1} \cup C_{3}$, il suffit de compter les lignes qui contiennent au moins un 1 dans la sous-matrice du Tableau 3.

\begin{tabular}{||l|l|}
\hline \hline 1 & 0 \\
\hline 0 & 0 \\
\hline 0 & 1 \\
\hline 1 & 0 \\
\hline 1 & 1 \\
\hline
\end{tabular}

TABLE 3 : Cardinalité de $C_{1} \cup C_{3}$.

Ainsi, $C_{M}\left(C_{1} \cup C_{3}\right)=4$.

La cardinalité $C_{M}\left(C_{1} \cap C_{2}\right)=1$. Elle est égale au nombre de lignes qui contiennent que des 1 dans la sous matrice du Tableau 4.

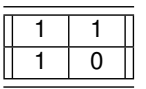

TABLE 4 : Cardinalité de $C_{1} \cap C_{2}$.

\section{Expérimentations}

Les images de télédétection présentent des caractéristiques spatiales, spectrales et temporelles différentes. Elles peuvent être acquises par une variété de capteurs ayant différentes résolutions. Dans ce contexte, la fusion peut s'appliquer afin de résoudre quelques difficultés telles que la détection des changements et la cartographie. Nous avons appliqué les théories des possibilités, des fonctions de croyance et du raisonnement plausible et paradoxal dans un cadre ponctuel (fusion bas niveau) en tenant compte de l'information spectrale pour réaliser la classification supervisée d'images très haute résolution d'une scène forestière prises par le satellite QuickBird en 2006.

Dans un premier temps, nous avons fusionné une première image très haute résolution (THR1) avec l'inventaire forestier correspondant (IF1). Par la suite, nous avons réalisé une deuxième expérience en fusionnant l'image QuickBird avec l'image de Hölder correspondante pour réaliser la classification texturale. Sachant que cette dernière donne une idée sur la régularité de la texture de la THR (Figure 5).

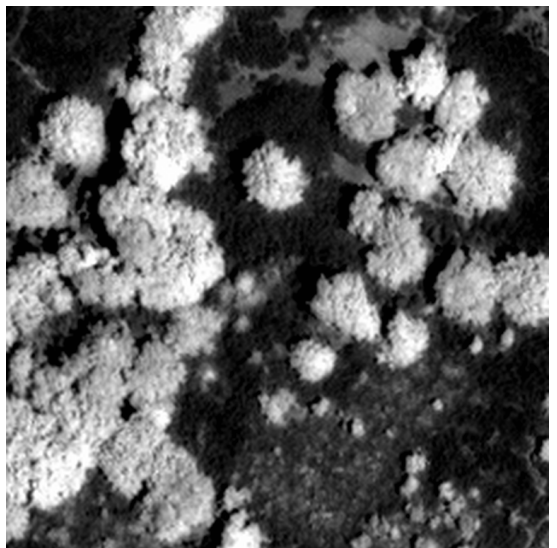

(1)

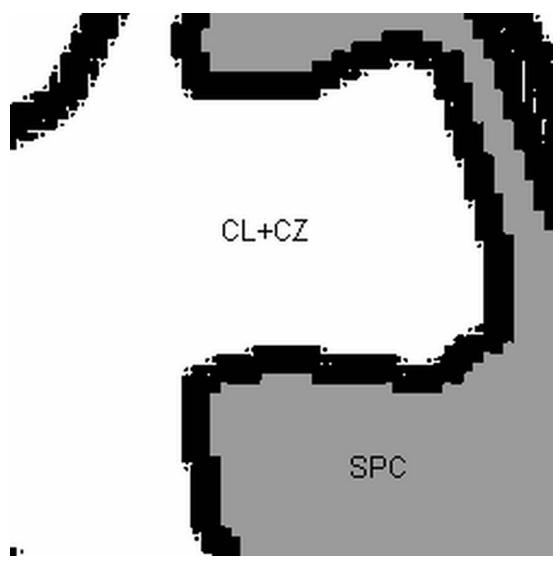

(2)

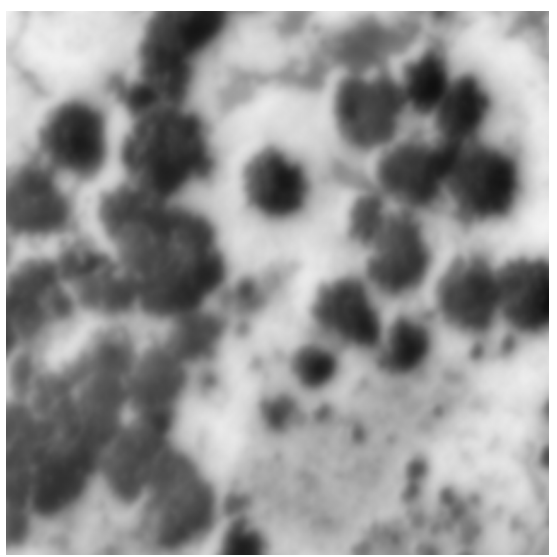

(3)

FIguRE 5 : (1) : Fenêtre 1 de l'image Quickbird THR, (2) : Inventaire forestier correspondant, (3) : Image de Hölder.

Les échantillons pris pour la classification ainsi que l'image de conflit entre la THR1 et l'IF1 sont illustrés par la figure 6 .

Les THR 1 et 2 sont formées par quatre classes qui sont : Chêne Zen (CZ), Chêne Liège $(C L)$, Sol Peu Couvert (SPC) et Sol $\mathrm{Nu}(\mathrm{SN})$. L'information fournie par les sources est entachée d'imprécision. En effet, les informations acquises par une source ne nous permettent pas de décider dans certains cas sur l'appartenance exacte d'un pixel à une classe donnée. De plus, la strate 


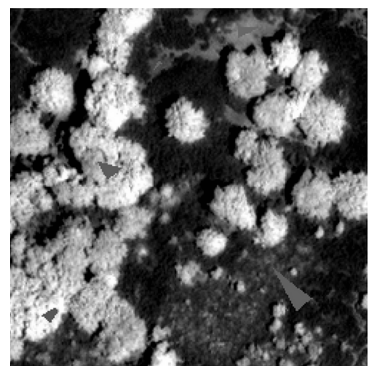

(1)

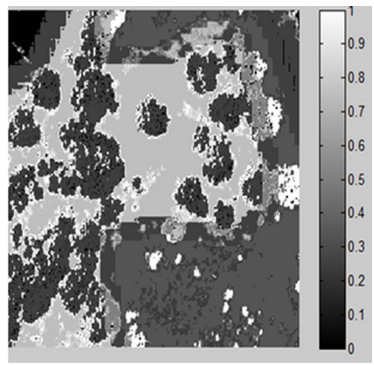

(2)
FIGURE 6 : (1) Échantillons, (2) Image de conflit entre la THR1 et l'IF1.

de " $C Z+C L$ " représente un mélange de chênes et donc elle symbolise plus qu'une classe. Cette nature imprécise de l'inventaire induit l'incertitude. Pour un pixel donné d'une image THR, nous disposons au niveau de l'inventaire d'un polygone de la région qui l'entoure et non une information spécifique. La nature imprécise et incertaine des sources favorise l'apparition du conflit. De plus, les sources n'ont pas le même référentiel de temps (image QuickBird 2006 , inventaire 2000), par conséquent des changements ont eu lieu surtout au niveau de la couverture forestière ce qui engendre l'ambiguïté et un conflit fort (pixels ayant un niveau de gris proche du blanc dans l'image de conflit).

Nous avons réalisé la classification d'une deuxième image de très haut résolution THR2 en la fusionnant avec l'inventaire forestier qui lui correspond.

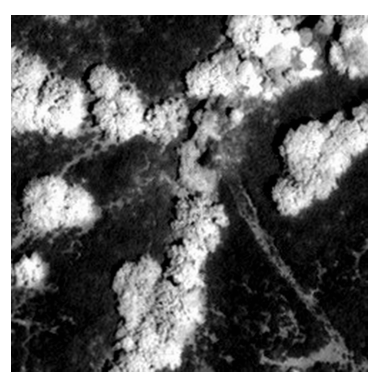

(1)

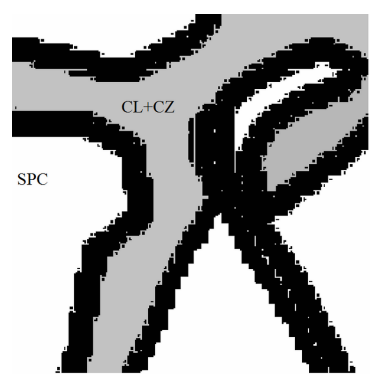

(2)
FIGURE 7 : (1) : Fenêtre 2 de l'image Quickbird THR (2) : Inventaire forestier correspondant.

\subsection{Fusion avec l'inventaire}

\subsubsection{Classification de THR1}

La THR et l'inventaire forestier ne présentent pas le même univers du discours ainsi on ne peut pas fusionner ces deux sources par la théorie des possibilités. Les résultats de la classification de la première image par la TFC et la DSmT sont illustrés par la figure 8.

Dans un premier temps, pour appliquer la TFC nous avons affaibli le jeu de masse de l'inventaire par un coefficient $\alpha=0,8$ afin de réduire le conflit entre les deux sources. L'analyse visuelle du résultat obtenu par la TFC

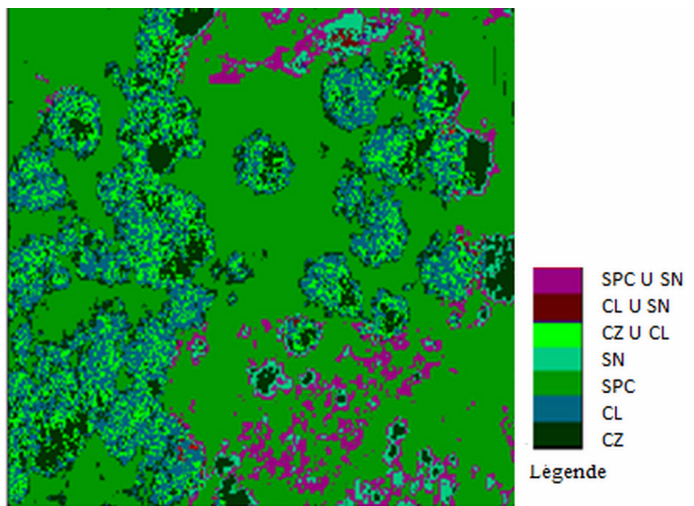

(a) Classification de THR1 par la TFC.

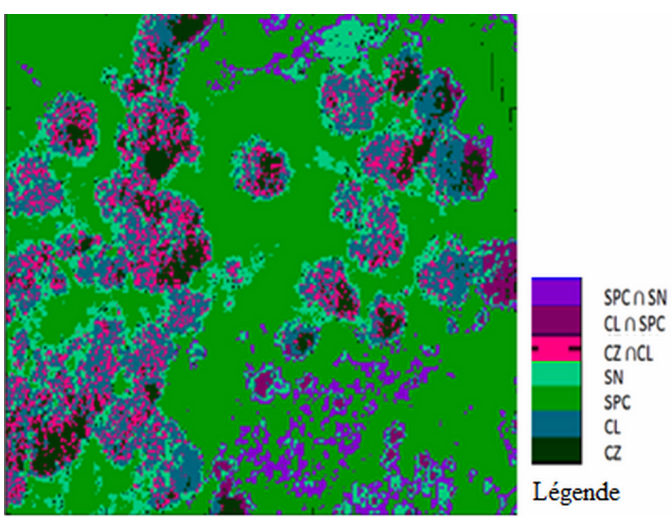

(b) Classification de THR1 par la DSmT.

FIgURE 8 : Résultats de classification par la TFC et la DSmT.

montre que cette dernière a mal classé des pixels conflictuels des classes $C L$ et $S P C$. Ces pixels sont affectés à la classe $C Z$. De même, les pixels du contour de la classe $C L$ avec la classe $S N$ sont affectés à la classe $C Z$ ce qui est faux. Par contre, la DSmT a correctement affecté ces pixels à la classe $S N$. Les pixels conflictuels entre les classes $C L$ et $S P C$ sont mis dans une classe paradoxale qui est $C L \cap S P C$. Les pixels affectés à la classe paradoxale $C L \cap S P C$ appartiennent à $C L$ dans la THR et à $S P C$ dans l'IFN. Les pixels mal classés par la TFC ont des niveaux de conflit élevés comme le montre la figure 9.

En appliquant la théorie des possibilités pour réaliser la classification de la THR1 sans la fusionner avec l'inventaire (classification mono-source), nous avons obtenu les résultats indiqués sur la figure 10. on remarque que dans l'image résultante, plusieurs pixels dispersés sont affectés improprement à la classe $C Z$ (vert foncé). II est clair que la classification mono-source est insuffisante pour gérer l'incertitude d'où l'intérêt de la fusion.

\subsubsection{Classification de THR2}

Les résultats de la classification par la TFC et la DSmT sont illustrés par la figures 11. dans cette expérience, nous pouvons constater de nouveau que le résultat de classification par la DSmT est nettement meilleur que celui obtenu par la TFC. Cette dernière a échoué encore 


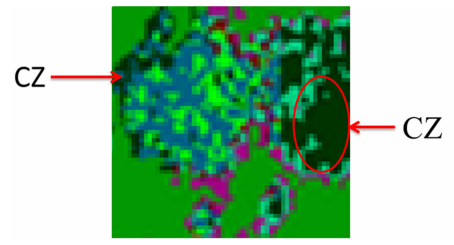

(1)

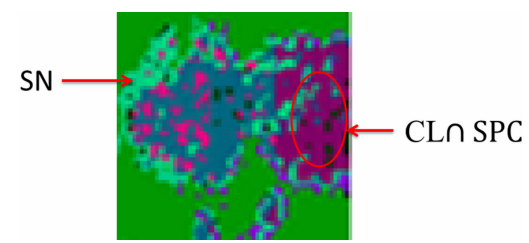

(2)

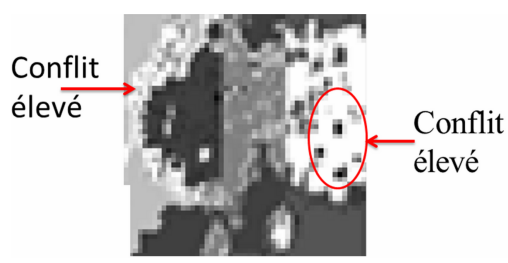

(3)

FIGURE 9 : Comparaison des résultats : (1) : TFC, (2) : DSmT, (3) : Pixels conflictuels.

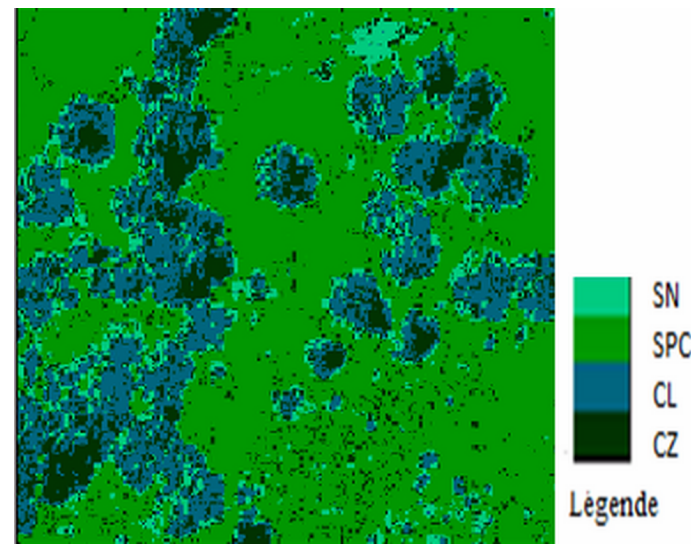

FIGURE 10 : Classification mono-source par la théorie des possibilités.

dans la classification des frontières entre les classes. En effet, encore un fois les pixels entre les classes $S P C$, $S N$ et $C L$ sont affectés improprement à la classe $C Z$. En outre, ces pixels sont affectés soit à $S P C$ soit à une classe composée. Les frontières de $C Z$ et $C L$ sont mises dans une classe paradoxale.

Les résultats obtenus par la DSmT prouvent, d'une part, l'efficacité de notre méthode proposée pour la construction des classes paradoxales et, d'autre part, ils confirment la capacité de cette théorie à fusionner des sources hautement conflictuelles et présentant une forte ambiguïté.

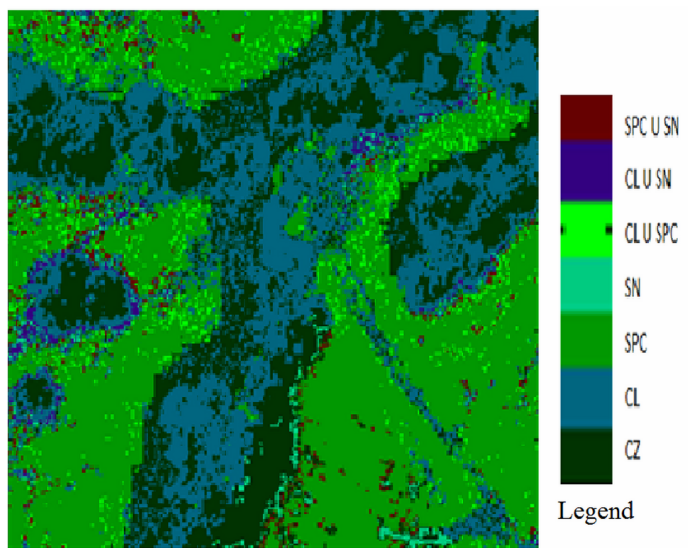

(a) Classification de THR2 par la TFC.

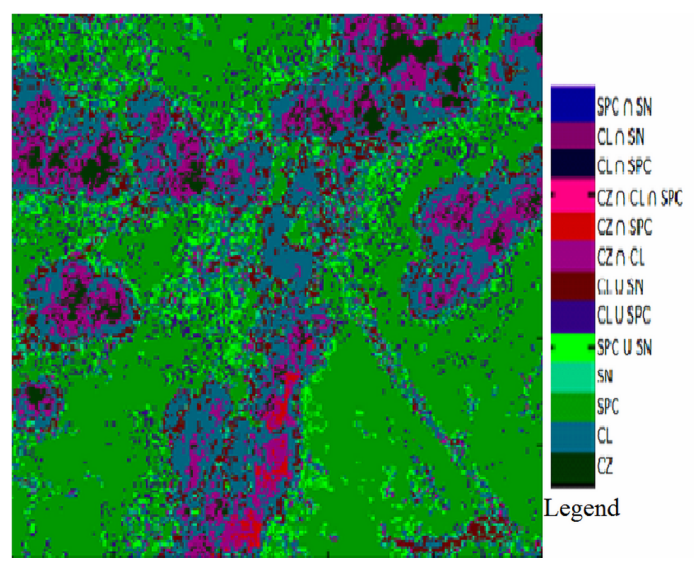

(b) Classification de THR2 par la DSmT.

FIgURE 11 : Résultats de classification de THR2 par la TFC et la DSmT.

\subsection{Fusion de THR1 avec l'image de Hölder}

Le résultat de la fusion par la TFC est obtenu à l'issue de l'application du critère de maximum de la probabilité pignistique qui nous permet d'avoir une image formée par les singletons. Nous avons choisi le critère de minimum d'incertitude (maximum de crédibilité) dans le cas de la DSmT.

L'analyse visuelle des résultats de la classification texturale ne nous permet pas de déterminer quelle est la meilleure classification. Pour cela, nous avons utilisé la matrice de confusion pour évaluer les résultats (Tableaux 5 et 6 ).

\begin{tabular}{||c|c|c|c|c||}
\hline \hline $\mathrm{CR}$ & $\mathrm{CZ}$ & $\mathrm{CL}$ & $\mathrm{SPC}$ & $\mathrm{SN}$ \\
\hline $\mathrm{CZ}$ & 100 & 0 & 0 & 0 \\
\hline $\mathrm{CL}$ & 16,0714 & 83.9286 & 0 & 0 \\
\hline $\mathrm{SPC}$ & 0 & 0 & 98,1884 & 1,8116 \\
\hline $\mathrm{SN}$ & 11,4754 & 0 & 1,6393 & 86,8852 \\
\hline
\end{tabular}

TABLE 5 : Matrice de confusion de la classification texturale par la TFC.

Où $\mathrm{CR}$ et $\mathrm{CE}$ représentent respectivement les classes réelles et les classes estimées. L'analyse des diagonales 


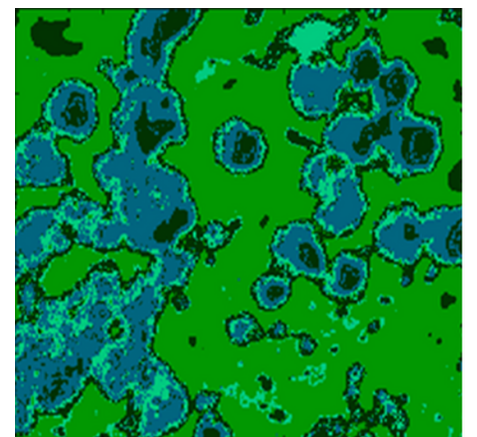

(1)

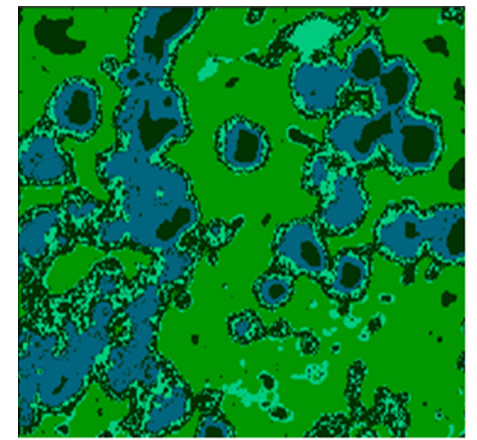

(2)

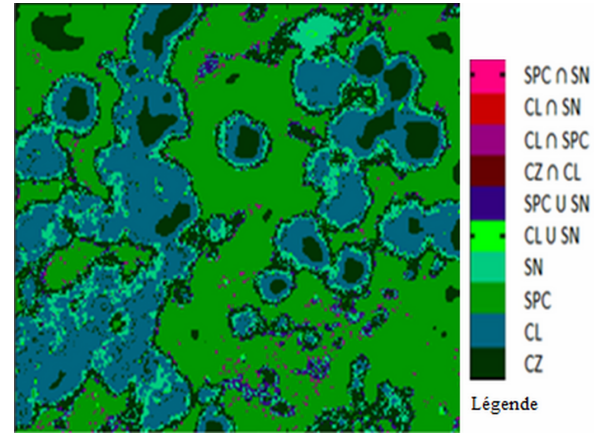

(3)

FIGURE 12 : (1) : Classification par la théorie des possibilités, (2) : Classification par la TFC, (3) : Classification par la DSmT.

\begin{tabular}{||c|c|c|c|c||}
\hline \hline $\mathrm{CR}$ & $\mathrm{CZ}$ & $\mathrm{CL}$ & $\mathrm{SPC}$ & $\mathrm{SN}$ \\
\hline $\mathrm{CZ}$ & 100 & 0 & 0 & 0 \\
\hline $\mathrm{CL}$ & 17,8571 & 82,1429 & 0 & 0 \\
\hline $\mathrm{SPC}$ & 0 & 0 & 98,9130 & 1,0870 \\
\hline $\mathrm{SN}$ & 12,2951 & 0 & 5,7377 & 81,9672 \\
\hline
\end{tabular}

TABLE 6 : Matrice de confusion de la classification texturale par la théorie des possibilités.

des matrices de confusion, ainsi que les indices Kappa $\left(\kappa_{T F C}=0,9041\right.$ et $\left.\kappa_{\text {poss }}=0,8874\right)$ confirment que les résultats obtenus par les classifications évidentielle et possibiliste sont satisfaisantes et légèrement différentes. En effet, pour l'approche possibiliste les taux de bonne classification des classes $C Z$ et $S P C$ sont assez élevés par rapport à ceux des classes $C L$ et $S N$. En comparant ces résultats à ceux obtenus par la classification par la TFC, nous constatons que les pourcentages de bonne

\begin{tabular}{||c|c|c|c|c|}
\hline \hline $\mathrm{CR}$ & $\mathrm{CZ}$ & $\mathrm{CL}$ & $\mathrm{SPC}$ & $\mathrm{SN}$ \\
\hline $\mathrm{CZ}$ & 100 & 0 & 0 & 0 \\
\hline $\mathrm{CL}$ & 16,0714 & 83,9286 & 0 & 0 \\
\hline $\mathrm{SPC}$ & 0 & 0 & 95,2899 & 0,3623 \\
\hline $\mathrm{SN}$ & 11,4754 & 0 & 0,8197 & 60,6557 \\
\hline
\end{tabular}

\begin{tabular}{|c|c|c|c|}
\hline$C L \cup S N$ & $S P C \cup S N$ & $C L \cap S P C$ & $C L \cap S P C \cap S N$ \\
\hline 0 & 0 & 0 & 0 \\
\hline 0 & 0 & 0 & 0 \\
\hline 0 & 1,4493 & 0 & 2,8986 \\
\hline 0,8197 & 21,3115 & 0,8197 & 4,0984 \\
\hline
\end{tabular}

TABLE 7 : Répartition des affectations sur les éléments focaux obtenus suite à la classification texturale par la DSmT.

classification de $C Z$ et $S P C$ sont quasi-identiques alors que les taux de classification des classes $C L$ et $S N$ sont meilleurs.

Les taux de bon classification par la TFC des classes $C Z$ et $C L$ et ceux de répartition par la DSmT sont identiques. De plus, les pourcentages d'affection aux classes $S P C$ et $S N$ par la DSmT sont inférieurs aux taux de bonne classification par les approches possibiliste et évidentielle de ces mêmes classes. Les différences sont affectées principalement à des classes composées comme le montre la matrice de répartition. Les deux images fusionnées ne présentent pas un conflit élevé ce qui justifie les résultats proches par les trois théories et valide l'étude théorique comparative.

\section{Conclusion}

L'objectif de ce travail est de comparer les théories des possibilités, des fonctions de croyance, du raisonnement plausible et paradoxal afin de pouvoir choisir la théorie la plus adéquate pour fusionner les sources disponibles. Le choix d'un formalisme de fusion est une étape déterminante puisqu'un choix non approprié engendre généralement des résultats non souhaités. La comparaison de ces théories est difficile puisque leurs modélisations, raisonnements et interprétations ne sont pas similaires et ceci peut revenir en premier lieu à leurs fondements mathématiques différents (base flou et probabiliste). La comparaison a donc été orientée vers les informations et leurs imperfections traitées par chaque théorie. Les trois approches modélisent les deux imperfections les plus étudiées qui sont l'incertitude et l'imprécision. Néanmoins, pour des sources hautement en conflit, la DSmT fournit le cadre théorique le plus convenable.

Nous avons validé l'étude théorique par une application de classification d'images de très haute résolution spatiale. Les sources impliquées sont conflictuelles et n'ont pas le même degré de fiabilité. Pour cela, trois processus de classification ont été mis en place correspondant aux trois approches numériques étudiées. Les résultats obtenus sont cohérents avec l'étude théorique réalisé et la stratégie proposée. 
Pour générer les classes paradoxales, nous avons utilisé les histogrammes de niveaux de gris en se concentrant sur les zones de confusion. La fusion des images THR avec les inventaires forestiers qui leur correspondent valorise cet apport. Cette méthode nous a également permis d'intégrer systématiquement des contraintes d'exclusivité. Ainsi, la cardinalité de l'hyperpuissance et la complexité temporelle sont réduites. Une autre méthode particulière est également proposée pour ordonner et coder les éléments focaux généralisés. En se basant sur cette dernière, nous avons trouvé une nouvelle méthode pour calculer la cardinalité de DSm ce qui représente l'intérêt (majeur) de la technique de codification.

\section{Références}

Alsun, M. H., Lecornu, L., Solaiman, B., Le Guillou, C., Cauvin, J. M., 2010. Medical diagnosis by possibilistic classification reasoning. In : Conference on Information Fusion (FUSION). IEEE, Edinburgh, Scotland, pp. 1-7.

Appriou, A., 2005. Approche générique de la gestion de l'incertain dans les processus de fusion multisenseur. Traitement du Signal 22 (4), 307-3019.

Ben Amor, S. B., Martel, J.-M., 2004. Le choix d'un langage de modélisation des imperfections de l'information en aide à la décision. In : Annual Conference of the Administrative Sciences Association of Canada.

Ben Dhiaf, Z., 2011. Fusion de sources d'information pour l'interprétation d'images de télédétection moyenne et haute résolution : Application à l'inventaire forestier. PhD thesis, Universitè de Tunis El Manar, Tunisia.

Bloch, I., 1996. Information combination operators for data fusion : A comparative review with classification. IEEE Transactions on Systems, Man and Cybernetics, Part A : Systems and Humans 26 (1), 52-67.

Bloch, I., 2005. Fusion d'informations numériques : panorama méthodologique. In : Journées Nationales de la Recherche en Robotique. Guidel, France, p. 79.

Bloch, I., Maître, H., 2004. Les méthodes de raisonnement dans les images. Lecture of Ecole Nationale Supérieure des Télécommunications-CNRS UMR 5141.

Borotschnig, H., Paletta, L., Pinz, A., 1999. A comparison of probabilistic, possibilistic and evidence theoretic fusion schemes for active object recognition. Computing 62 (4), 293-319.

Destercke, S., Dubois, D., Chojnacki, E., 2006. Fusion d'opinions d'experts et théories de l'incertain. In : Proc. Rencontres Francophones sur la logique floue et ses applications. Toulouse, France.

Dezert, J., 2002. Foundations for a new theory of plausible and paradoxical reasoning. Information and Security 9, 13-57.

Dezert, J., Smarandache, F., 2003. On the generation of hyperpowersets for the DSmT. In : Conference on Information Fusion (FUSION). Istanbul, Turkey.

Dezert, J., Smarandache, F., 2005. The DSmT approach for information fusion and some open problems. In : NATO Advanced Study Institute. Albena, Bulgaria.

Dezert, J., Smarandache, F., 2009. An introduction to DSmT. arXiv preprint arXiv :0903.0279.

Dezert, J., Smarandache, F., Daniel, M., 2004. The generalized pignistic transformation. In : Conference on Information Fusion (FUSION). Stockholm, Sweden.
Dubois, D., Prade, H., 1985. Théorie des possibilités. Applications et Représentation des Connaissances en Informatique. Collection Méthode+ Programmes, Masson, Paris, France.

Dubois, D., Prade, H., 1994a. La fusion d'informations imprécises. Traitement du signal 11 (6), 447-458.

Dubois, D., Prade, H., 1994b. Possibility theory and data fusion in poorly informed environments. Control Eng. Practice 2, 811-823.

Dubois, D., Prade, H., 2003. Possibility theory and its applications : a retrospective and prospective view. In : IEEE International Conference on Fuzzy Systems. Vol. 1. IEEE, SaintLouis, MO, USA, pp. 5-11.

Dubois, D., Prade, H., 2009. Formal representations of uncertainty. Decision-Making Process : Concepts and Methods, 85-156.

Florea, M. C., Jousselme, A.-L., Bossé, I., Grenier, D., Apr. 2009. Robust combination rules for evidence theory. Information Fusion 10 (2), 183-197.

Khaleghi, B., Khamis, A., Karray, F. O., Razavi, S. N., 2011. Multisensor data fusion: A review of the state-of-the-art. Information Fusion 14 (1), 8-44.

Lefevre, E., Colot, O., Vannoorenberghe, P., 2002. Belief functions combination and conflict management. Information $\mathrm{Fu}$ sion Journal 3 (2), 149-162.

Li, X., Huang, X., Wang, M., 2006. Robot map building from sonar sensors and dsmt. Information \& Security Journal, Bulg. Acad. of Sci., Sofia 20.

Liu, W., 2007. Conflict analysis and merging operators selection in possibility theory. In : European Conferences on Symbolic and Quantitative Approaches to Reasoning with Uncertainty, ECSQARU. Hammamet, Tunisia, pp. 816-827.

Martin, A., 2005. La fusion d'informations. ENSIETA TechReport $1484,117$.

Martin, A., 2008. Fusion de classifieurs pour la classification d'images sonar. In : EGC. Sophia-Antipolis, France.

Martin, A., 2010. Le conflit dans la théorie des fonctions de croyance. In : EGC. Hammamet, Tunisia, pp. 655-666.

Milisavljevic, N., Bloch, I., 2003. Sensor fusion in anti-personnel mine detection using a two-level belief function model. IEEE Transactions on Systems, Man, and Cybernetics, Part C : Applications and Reviews 33 (2), 269-283.

Parsons, S., Hunter, A., 1998. A review of uncertainty handling formalisms. In : Applications of uncertainty formalisms. Springer, pp. 8-37.

Roux, L., Desachy, J., 1995. Information fusion for supervised classification in a satellite image. In : IEEE International Conference on Fuzzy Systems. IEEE, Yokohama, Japan, pp. 1119-1124.

Shafer, G., 1976. A mathematical theory of evidence. Princeton University Press, USA.

Shafer, G., 1990. Perspectives on the theory and practice of belief functions. International Journal of Approximate Reasoning 4 (5), 323-362.

Shafer, G., Logan, R., 1987. Implementing Dempster's rule for hierarchical evidence. Artificial Intelligence 33, 271-298.

Smarandache, F., Dezert, J., 2005a. An introduction to the DSm theory for the combination of paradoxical, uncertain, and imprecise sources of information. In : International Congress of Cybernetics and Systems. Maribor, Slovenia.

Smarandache, F., Dezert, J., 2005b. Proportional conflict redistribution rule. Journal of Advances in Information Fusion 1.

Smarandache, F., Dezert, J., 2006. Advances and applications of DSmT for information fusion : collected works. Vol. 2. American Research Press.

Smarandache, F., Dezert, J., Tacnet, J., 2010. Fusion of sources of evidence with different importances and reliabilities. In : Conference on Information Fusion. IEEE, Edinburgh, Scot- 
land, pp. 1-8.

Smets, P., 1990a. Constructing the pignistic probability function in a context of uncertainty. In : Uncertainty in Artificial Intelligence. Cambridge, MA, USA.

Smets, P., 1990b. The transferable belief model and other interpretations of Dempster-Shafer's model. In : Uncertainty in Artificial Intelligence. Cambridge, MA, USA.

Smets, P., 1997. Imperfect information : Imprecision and uncertainty. In : Uncertainty Management in Information Systems. Springer, Providence, RI, USA.

Smets, P., 1998. Probability, possibility, belief : which and where. Handbook of defeasible reasoning and uncertainty management systems 1, 1-24.

Tchamova, A., Dezert, J., 2012. On the behavior of dempster's rule of combination and the foundations of Dempster-Shafer Theory. In : IEEE International Conference Intelligent Systems. IEEE, Sofia, Bulgaria, pp. 108-113.

Vannoorenberghe, P., 2003. Un état de l'art sur les fonctions de croyance appliquées au traitement de l'information. Revue I3 3 (2), 9-45.

Zadeh, L. A., 1968. Fuzzy algorithms. Information and control 12, 94-102.

Zadeh, L. A., 1978. Fuzzy sets as a basis for a theory of possibility. Fuzzy Sets and Systems 1 (1), 3-28.

Zadeh, L. A., 1984. Book review : A mathematical theory of evidence. Al Magazine 5 (3), 81-83. 